\title{
Prediction of Revascularization after Myocardial Perfusion SPECT by Machine Learning in a Large Population
}

\author{
Reza Arsanjani, $\mathbf{M D}^{1,3}$, Damini Dey, $\mathrm{PhD}^{1,3}$, Tigran Khachatryan, $\mathbf{M D}^{1}$, Aryeh Shalev, MD ${ }^{1}$, \\ Sean W. Hayes, MD1 , Mathews Fish, MD², Rine Nakanishi, MD ${ }^{1}$, Guido Germano, PhD $^{1,3}$, \\ Daniel S. Berman, $\mathbf{M D}^{1,3}$, and Piotr Slomka, PhD ${ }^{1,3}$ \\ ${ }^{1}$ Departments of Imaging and Medicine, and Cedars-Sinai Heart Institute, Cedars-Sinai Medical \\ Center, Los Angeles, CA \\ 2 Oregon Heart and Vascular Institute, Sacred Heart Medical Center, Springfield, OR \\ ${ }^{3}$ David Geffen School of Medicine, University of California Los Angeles, Los Angeles, CA
}

\section{Abstract}

Objective-We aimed to investigate if early revascularization in patients with suspected coronary artery disease (CAD) can be effectively predicted by integrating clinical data and quantitative image features derived from perfusion SPECT (MPS) by machine learning (ML) approach.

Methods-713 rest ${ }^{201}$ Thallium/stress ${ }^{99 \mathrm{~m}}$ Technetium MPS studies with correlating invasive angiography (372 revascularization events (275 PCI / 97 CABG) within 90 days after MPS (91\% within 30 days) were considered. Transient ischemic dilation (TID), stress combined supine/prone total perfusion deficit (TPD), quantitative rest and stress TPD, exercise ejection fraction, and endsystolic volume along with clinical parameters including patient gender, history of hypertension and diabetes mellitus, ST-depression on baseline ECG, ECG and clinical response during stress, and post-ECG probability by boosted ensemble ML algorithm (LogitBoost) to predict revascularization events. These features were selected using an automated feature selection algorithm from all available clinical and quantitative data (33 parameters). 10-fold cross-validation was utilized to train and test the prediction model. The prediction of revascularization by ML algorithm was compared to standalone measures of perfusion and visual analysis by two experienced readers utilizing all imaging, quantitative, and clinical data.

Results-The sensitivity of machine learning $(73.6 \pm 4.3 \%)$ for prediction of revascularization was similar to one reader $(73.9 \pm 4.6 \%)$ and standalone measures of perfusion $(75.5 \pm 4.5 \%)$. The specificity of machine learning $(74.7 \pm 4.2 \%)$ was also better than both expert readers $(67.2 \pm 4.9 \%$ and $66.0 \pm 5.0 \%, \mathrm{P}<0.05)$, but was similar to ischemic TPD $(68.3 \pm 4.9 \%, \mathrm{P}<0.05)$. The ReceiverOperator-Characteristics areas-under-curve for machine learning $(0.81 \pm 0.02)$ was similar to reader $1(0.81 \pm 0.02)$ but superior to reader $2(0.72 \pm 0.02, \mathrm{P}<0.01)$ and standalone measure of perfusion $(0.77 \pm 0.02, \mathrm{P}<0.01)$.

Corresponding Author Info: Piotr J. Slomka, PhD, Artificial Intelligence in Medicine Program, 8700 Beverly Blvd, Ste A047N, Los Angeles, CA 90048, USA, Ph: 310-423-4348, piotr.slomka@ cshs.org.

Reza Arsanjani, MD, Cedars-Sinai Medical Center, 8700 Beverly Blvd, Taper A238, Los Angeles, CA 90048, USA, Ph:

310-248-7707, Reza.Arsanjani@cshs.org 
Conclusion-ML approach is comparable or better than experienced reader in prediction of the early revascularization after MPS and is significantly better than standalone measures of perfusion derived from MPS.

\section{Keywords}

Machine Learning; Coronary Artery Disease; Myocardial Perfusion SPECT; Revascularization; Total Perfusion Deficit

\section{Introduction}

Over the last three decades, myocardial perfusion imaging with SPECT (MPS) has been the most commonly utilized noninvasive imaging technique in the low to intermediate risk population for assessing the presence of obstructive coronary artery disease (CAD) ${ }^{1}$. Prior studies have demonstrated that myocardial perfusion is reduced in the presence of a $\geq 70 \%$ epicardial luminal stenosis ${ }^{2}$, and MPS has excellent sensitivity and specificity for the detection of angiographically significant (defined as more than $70 \%$ stenosis) $\mathrm{CAD}^{1}$. Therefore, invasive coronary angiography is often used as the gold standard for assessing the diagnostic accuracy of MPS for predicting presence of obstructive coronary artery disease. However, the ability of MPS to predict revascularization has not been previously studied.

The interpretation of MPS is currently performed primarily by experienced clinicians who manually combine raw and perfusion images, incorporate quantitative, functional, and clinical data in a systematic fashion based on the American Society of Nuclear Cardiology (ASNC) recommendations ${ }^{3}$. However, the interpretation is mostly semi-quantitative, subjective, and heavily dependent on reader's experience level ${ }^{4}$. Prior studies have evaluated multiple MPS features, including semi-quantitative and quantitative perfusion and functional variables, for diagnosis of obstructive $\mathrm{CAD}^{5-9}$. In addition, prior studies have demonstrated that standalone quantitative perfusion parameters compare favorably to visual scoring for diagnosing obstructive $\mathrm{CAD}^{10}$. Furthermore, the use of machine learning (ML) algorithms for combining and integrating perfusion, functional, and clinical variables for diagnosis of obstructive CAD allows one to obtain results similar or better than those obtained by experienced readers ${ }^{11}$. However, combining these parameters by ML for predicting revascularization events has not been previously studied.

In this study, we aimed to investigate if early revascularization in patients with suspected coronary artery disease (CAD) can be effectively predicted by integrating clinical data and quantitative image features derived from perfusion SPECT (MPS) by boosting ML algorithm (LogitBoost), which is the modern ML method, based on additive modeling of maximum likelihood ${ }^{12}$.

\section{Materials and Methods}

\section{Patient Population}

The subjects with suspected CAD who were referred to the Nuclear Medicine Department of Cedars-Sinai Medical Center, Los Angeles, California, from November 1, 2001 to June 30, 2005 for rest and stress electrocardiography (ECG)-gated, performed in both supine and 
prone positions, were consecutively selected ${ }^{11}$. Consecutive patients in whom MPS and coronary angiography with or without revascularization were performed within 60 days, without a significant intervening event were studied. All patients with a prior history of CAD, known cardiomyopathy, left bundle branch block, pacemaker, or significant valve disease were excluded. Based on these criteria, 713 sequential studies were identified to form the patient population, which constituted approximately $5 \%$ of all consecutive MPS studies performed at that period. The clinical characteristics are listed in Table 1. The data analyzed in this study have been obtained retrospectively from the existing database at Cedars-Sinai Medical Center (Los Angeles, California). The retrospective use of clinical data in this study was approved by the Institutional Review Board at Cedars-Sinai and informed consent was obtained from all patients.

\section{Image Acquisition and Reconstruction Protocols}

All patients underwent separate-acquisition rest ${ }^{201} \mathrm{Tl} /$ stress ${ }^{99 \mathrm{~m}} \mathrm{Tc}$-sestamibi dual-isotope MPS as previously described ${ }^{13}$. Images were acquired on a dual-detector camera (Forte or Vertex [Philips Medical Systems, Andover, Massachusetts] or e.cam [Siemens Medical Systems, Malvern, Pennsylvania]) equipped with a high-resolution collimator. The details of image acquisition and tomographic reconstruction have been previously described ${ }^{14}$.

Briefly, for rest imaging, weight- adjusted ${ }^{201} \mathrm{Tl}(3.0-4.5 \mathrm{mCi})$ was injected intravenously and acquisition was performed 10 minutes after injection, with 35 seconds per projection. In patients able to exercise, a symptom limited exercise treadmill test with the standard Bruce protocol was performed. Treadmill exercise was continued at maximum workload for 1 minute and at one stage lower for 2 additional minutes when possible, ${ }^{99 \mathrm{~m}} \mathrm{Tc}$-sestamibi MPS acquisition was started 15 to 30 minutes after radiopharmaceutical injection. In patients undergoing pharmacological stress testing, adenosine was infused at $140 \mu \mathrm{g} \cdot \mathrm{kg}-1 \cdot \mathrm{min}^{-1}$ for 5 minutes ${ }^{15}$. At the end of the second minute, ${ }^{99} \mathrm{~m} \mathrm{Tc}$-sestamibi $(25-40 \mathrm{mCi}$ based on patient's weight) was injected. Whenever possible, during adenosine infusion, patients performed a low-level treadmill exercise, walking at $0 \%$ grade at 1 to $1.7 \mathrm{mph}^{16}$. Poststress ${ }^{99 \mathrm{~m}} \mathrm{Tc}$-sestamibi acquisitions were performed beginning 15 to 60 minutes after injection, with 64 projections at 25 seconds per projection for supine ${ }^{99 \mathrm{~m}} \mathrm{Tc}$ acquisition, followed immediately by 32 projections at 15 seconds per projection for prone ${ }^{99 \mathrm{~m}} \mathrm{Tc}$ acquisition. No attenuation or scatter correction was applied.

Studies were reconstructed on the respective vendor platforms (Pegasys [Philips Medical Systems] or e.soft 2000 [Siemens Medical Systems]) by use of their commercial implementations of the iterative reconstruction. The reconstruction parameters were 12 iterations with Butterworth prefiltering (cutoff, 0.66 cycles per pixel for supine ${ }^{99 \mathrm{~m}} \mathrm{Tc}$ and 0.55 cycles per pixel for prone ${ }^{99 \mathrm{~m}} \mathrm{Tc}$; order, 5 ). These parameters were optimized previously to provide optimal image quality for clinical scan reading ${ }^{17}$. Gated images were only obtained for the supine position. Short-axis images were automatically generated ${ }^{18}$. In certain cases when the software failed to detect left ventricular contours, manual and visual assessment was needed for the derivation of the automated results by an experienced technologist. In addition, if patient motion was noted during review of raw projections, 
motion correction (MoCo) software was applied ${ }^{19}$. After reconstruction, the images were transferred for visual analysis using Cedars-Sinai QPS software ${ }^{20}$.

\section{Clinical Parameters}

We utilized multiple clinical features including age, gender, diabetes mellitus, hyperlipidemia, tobacco abuse, hypertension, claudication symptoms, family history of $\mathrm{CAD}$, weight, body mass index, typicality of symptoms. We also calculated the probability of obstructive CAD using ECG Diamond-Forrester criteria based on the degree of STdepression on stress $\mathrm{ECG}^{21}$ as well as vital signs during stress. All these clinical features were considered as the input to the automated feature selection algorithm.

\section{Image Parameters}

Several quantitative perfusion and function parameters were obtained in a fully automated mode from the MPS images. These parameters were also considered as an input to the ML algorithm. Supine and prone images were quantified separately using their respective supine and prone normal limits and a previously developed simplified approach ${ }^{7}$. The combined supine/prone TPD parameter, based on the findings of abnormality on both supine and prone images was computed as previously described ${ }^{14}$. Briefly, an optimal normalization factor between the database case and test-case was established by an iterative search and an abnormality threshold of 3.0 average (mean absolute) deviations was applied, which is approximately equivalent to $2.5 \mathrm{SD}$, to estimate the extent of hypoperfusion. The total perfusion deficit (TPD) measure was computed as the integral of polar map severities below the abnormality threshold, reflecting both extent and severity of the defect. In addition to deriving separate TPD measures for supine and prone MPS, we computed the combined supine/prone TPD parameter, based on the findings of abnormality on both supine and prone images as previously described ${ }^{14}$. Briefly, combined supine/prone TPD is calculated by limiting the TPD computation from the supine polar map to pixels, which have been quantified as abnormal on prone images. The same average deviation threshold (3.0) is used for supine and prone images (as in the analysis of separate images). The combined supine/ prone parameter was expressed in the same units (percentage of the myocardium) as in the separate supine and prone TPD measures. A stress TPD+ value of $>3 \%$ and ischemic TPD+ value of $>2 \%$ was considered abnormal. We refer to these combined TPD parameters as TPD in the remainder of this manuscript. Quantitative stress and rest perfusion deficits were derived as previously described ${ }^{6}$. Standard MPS processing was performed by the QPS/QGS software ${ }^{18,20}$. The TID ratio was derived from stress/rest supine MPS as previously defined ${ }^{23}$.

\section{Visual Analysis}

Visual interpretation of MPS images was based on short axis, horizontal and vertical longaxis slices, as well as ability to utilize gated images. The myocardium was divided into 17 segments using QPS interactive 17 -segment graph ${ }^{20}$ according to the American Heart Association system ${ }^{24}$. MPS images were scored independently by two expert cardiologists using a five-point scoring system (0-normal; 1-mildly decreased uptake; 2-moderately decreased uptake; 3 -severely decreased uptake; and 4-absence of segmental uptake). Visual reading was performed by expert readers with all data available including stress and rest 
perfusion data, raw projection data, and gated functional data including gated images and stress plus rest ejection fraction (EF), quantitative computer results, and all clinical information including age, cardiac risk factors, type of stress, and clinical and ECG responses to stress. Subsequently, summed stress scores (SSS) and summed rest scores were calculated by summing the 17 -segment stress and rest scores, respectively. A visual SSS score of $\geq 4$ and a visual summed difference scores (SDS) of $\geq 2$ was considered as a predictor or revascularization ${ }^{17}$.

\section{Machine learning methods}

\section{Feature Selection}

We utilized an automated feature selection algorithm, which evaluates the worth of an attribute by measuring the entropy gain with respect to the outcome and ranks the attributes by their individual evaluations ${ }^{25}$. The automated feature selection methods are a part of WEKA machine learning environment ${ }^{26}$. Only the attributes that were resulting in the information gain $>0$ were subsequently used as an input for the Logitboost classification algorithm. This is a standard machine learning procedure performed to ensure appropriate performance of the classification algorithm. As input, we utilized all the clinical features mentioned above as well as imaging quantitative parameters including TID, rest and stress combined supine/prone TPD, ejection fraction, left ventricular volumes, and quantitative stress/rest summed scores.

\section{Classification}

We have employed an ensemble-boosting LogitBoost algorithm, which is a meta-algorithm technique. A higher classification performance is obtained by combining several simple classification schemes, which produce a strong ensemble classification scheme by iteratively adjusting appropriate weights for each of the base-level classifiers. We utilized a LogitBoost procedure $^{12}$ with decision stumps as the base classifiers implemented in the Waikato Environment for Knowledge Analysis (WEKA) ${ }^{26}$. This method has previously been utilized for prediction of obstructive CAD from MPS ${ }^{11}$. A total of 33 attributes were utilized (Table 2). Ten-fold cross-validation (CV) technique was used to assess and compare the performance of the prediction by logistic regression models which ensured that none of the image data used for creation of logistic regression models was used in subsequent evaluation of the diagnostic performance of the same model ${ }^{27}$. Therefore, 10 different models were used to derive the final test results ${ }^{28}$. The use of 10 -fold $\mathrm{CV}$ allows for the construction of stable logistic regression models trained on $90 \%$ fractions of the population as well as allow us to evaluate the model variability. This technique has been shown to have the smaller bias for discriminant analysis than other methods such as split-sample (1). The classification output in the form of continuous probability estimates (from 0 to 1 ) was used to construct the receiver operator characteristic (ROC) curves, utilizing the 10-fold cross-validation results. The ML algorithm performance was compared to the expert visual readings and to the standalone perfusion parameters including stress TPD and ischemic TPD. Machine learning model without the features obtained from the resting scan (resting TPD, TID) was also considered to compare this approach to the visual and standard quantification prediction of the revascularization from stress-imaging alone combined with the clinical data. 


\section{Conventional Coronary Angiography / Revascularization}

Conventional invasive coronary angiography (ICA) was performed according to standard clinical protocols, within 60 days of the myocardial perfusion examination. Three-hundred and seventy-two out of 713 patients underwent revascularization also within the 60 days after the MPS, with 275 patients undergoing percutaneous coronary intervention (PCI) [75 with multi-vessel $\mathrm{PCI}]$ and 97 patients undergoing coronary artery bypass graft (CABG) surgery (7 with single-vessel CABG).

\section{Statistical Analysis}

Continuous variables were expressed as the mean \pm standard deviation, and categorical variables were expressed as percentages (\%). Z-test for comparison of proportions and McNemar was performed to compare the sensitivity and specificity. The stress TPD was compared to SSS, while ischemic TPD was compared to SDS. For all analyses, P values <. 05 were considered statistically significant. ROC curves were analyzed to evaluate the ability of ML software vs. visual scoring and standalone perfusion parameters to predict the early revascularization procedure. The differences between the ROC areas under the curves (AUC) were compared using the Delong method ${ }^{29}$.

\section{Results}

\section{Feature selection}

Thirteen total parameters were selected using the information gain ranking criteria including quantitative parameters including TID, stress combined supine/prone TPD, quantitative rest and stress TPD, exercise ejection fraction, and end-systolic volume along with clinical parameters including patient gender, history of hypertension and diabetes mellitus, STdepression on baseline ECG, ECG and clinical response during stress (chest pain with exercise), and post-ECG probability of CAD based on Diamond-Forrester criteria ${ }^{21}$ [Table 2]. The rankings in table 2 reflect the importance of each parameter.

\section{Machine Learning Versus Stress Visual Scores and Stress Standalone Perfusion}

The sensitivity of stress only machine learning for predicting revascularization $(72.7 \pm 4.5 \%)$ was similar to both readers' visual stress scores $(76.3 \pm 4.6 \%$ and $72.3 \pm 4.6 \%)$ and stress TPD $(77.7 \pm 4.5 \%)$ [Figure $1 \mathrm{~A}$ ]. The specificity of machine learning was similar to both readers' visual, but was significantly better than stress TPD $(74.5 \pm 4.1 \%$ vs. $67.4 \pm 5.0 \%, \mathrm{P}<0.05)$. The AUC for machine learning $(0.81 \pm 0.02)$ was similar to reader $1(0.81 \pm 0.02)$, but was significantly better than reader 2 and stress TPD $(0.76 \pm 0.02$ and $0.79 \pm 0.02$, respectively $(\mathrm{P}<$ $0.01)$ ) [Figure 2A]. We also assessed the likelihood of revascularization if the stress only machine probability score was greater than $75 \%$ versus less than $25 \%$. Eighty-four percent of patients (202) with a probability score of $>75 \%$ underwent revascularization, while only $16 \%$ of patients (171) with a probability score of $<25 \%$ underwent revascularization ( $\mathrm{P}<$ $0.001)$. 


\section{Machine Learning Versus Ischemic Visual Scores and Ischemic Standalone Perfusion}

The sensitivity of machine learning for predicting revascularization was significantly lower than reader $1(73.6 \pm 4.3 \%$ vs. $81.5 \pm 4.6 \%, \mathrm{P}<0.05)$, but was similar to reader 2 and ischemic TPD (Figure 1B). The specificity of machine learning was better than both readers $(74.7 \pm 4.2 \%$ vs. $67.2 \pm 4.9 \%$ and $66.0+5.0 \%, \mathrm{P}<0.05)$, but was similar to ischemic TPD. The ROC-AUC for machine learning $(0.81 \pm 0.02)$ was similar to reader $1(0.81 \pm 0.02)$, but was significantly better than reader 2 and ischemic TPD $(0.72 \pm 0.02$ and $0.77 \pm 0.02$, respectively $(\mathrm{P}<0.01)$ [ [Figure 2B]. We also assessed the likelihood of revascularization if stress/rest machine probability score was greater than $75 \%$ versus less than $25 \%$ (we selected quartiles for comparison). Eighty-four percent of patients (216) with probability score of $>75 \%$ underwent revascularization, while only $17 \%$ of patients (174) with probability score of $<25 \%$ underwent revascularization $(\mathrm{P}<0.001)$.

\section{Discussion}

In our current study, we were able to show that the machine algorithm combining clinical and quantitative MPS features was superior to standalone perfusion methods and at least comparable to experienced readers from high volume center in identifying individuals who will need to undergo revascularization. Thus, such ML software may hypothetically enable the physician to identify individuals who might benefit from invasive coronary angiography (because they are likely to undergo PCI as a part of invasive angiography procedure) as opposed to undergo only medical therapy. To our knowledge, this is a first study to evaluate an ability to computationally predict which patients would undergo revascularization after MPS. A recent study by Patel et al. demonstrated that only a third of the 400,000 patients studied had obstructive $\mathrm{CAD}$ at the time of invasive angiography and patients with positive noninvasive test were moderately more likely to have obstructive $\mathrm{CAD}^{30}$. Given this transformative period in healthcare when clinicians are expected to consistently foster better health outcomes at a lower cost, such automated algorithms may have the potential to reduce the need for additional downstream invasive testing, thus assisting physicians to adapt to the changing industry.

It should additionally be noted that the two readers in our study were from high volume centers, and prior studies have demonstrated that the degree of variability in MPS interpretation is dependent on the reader's experience ${ }^{31,32}$. It is therefore feasible that ML algorithms, which can easily be incorporated into currently available MPS software, might be even more useful for less experienced readers. Another important aspect of the machine learning is that in addition to classifying MPS as normal versus abnormal it also provides probability scores. We demonstrated that patients were 5 times more likely to undergo revascularization if they were in the highest quartile versus lowest quartile. These probability scores could be also be implemented in the currently available software, and could be used to assist the clinician when deciding who should be referred for invasive coronary angiography as well as revascularization. As demonstrated in table 2, the model was dominated by perfusion parameters; however clinical parameters also contributed to the model which highlights the benefits of using ML algorithms. 
The importance of abnormal MPS for predicting who might benefit most from revascularization has previously been evaluated. Hachamovitch and colleagues compared the short-term survival benefits associated with revascularization compared with medical therapy in 10,627 patients with no prior CAD undergoing MPS $^{33}$. They noted a greater survival benefit associated with revascularization in patients with moderate to large inducible ischemia on MPS studies (greater than 10\%). In addition, the benefits of medical therapy and revascularization based on extent of ischemia and scar were compared in 13,969 undergoing MPS ${ }^{34}$. Patients with significant ischemia and without extensive scar were more likely to benefit from early revascularization, while those with minimal ischemia had better outcomes with medical therapy. Although our study did not identify patients that might benefit most from revascularization, similar machine learning methodology could be used to compare the benefits of optimal medical therapy versus revascularization, if mortality is used as an outcome event as opposed to revascularization.

This study has several limitations. Revascularization was used as the gold standard for this study with its known limitations which were mentioned above. Attenuation correction was not used in this study; however, we used combined supine/prone analysis to guard against image artifacts. We have utilized supine-prone imaging protocols which is a clinical standard at our center but it is not routinely used in the majority of imaging centers, for both quantitative analysis and expert visual reading. Additionally, the MPS protocol was dualisotope imaging, which is limited by the difficulties in comparing rest and stress images due to differences in image resolution. This protocol has been employed less frequently recently due to the high amount radiation to the patient. Future studies using single-isotope protocols or low-dose MPS may be required to further analyze the ML algorithms for predicting revascularization. Nevertheless, we have studied patients with suspected CAD and also built the model utilizing only stress perfusion data, which demonstrated similar predictive performance to the model with stress and rest data. Thus, rest imaging data was not crucial in this analysis. The ML model was based on the global perfusion abnormalities rather than regional. In the future, we plan to apply these findings to individual coronary territories for assessment of localization of disease using ML. Another limitation of our current study is that it was conducted on retrospective fashion. It is difficult to assess how much the revascularization decision was biased by abnormal MPS studies, since the decision to revascularize could be affected by clinical MPS report. Nevertheless, the machine learning utilized objective quantitative parameters, which were not used in revascularization decisions. Furthermore, the visual analysis in our study was performed retrospectively in a blinded fashion and hence the obtained result would not affect the decision to vascularize. Finally, although we had a large patient population, the results were obtained in only one center. Further multicenter evaluations will be required to confirm these results.

\section{Conclusion}

A machine learning algorithm integrating clinical and quantitative MPS information is comparable or better than the experienced readers and is significantly better than standalone measures of perfusion derived from MPS, in predicting early revascularization following MPS. This methodology may allow reduction in unnecessary catheterization after MPS. 


\section{New Knowledge Gained}

Machine learning algorithms were used to integrate clinical and quantitative MPS imaging features and predict early revascularization events. This approach was evaluated in a large population of patients with suspected CAD undergoing MPS with correlative invasive coronary angiography. The automated computer algorithm was able to predict which patients would undergo revascularization better than standalone measures of perfusion derived from MPS and similar to experienced readers.

\section{Acknowledgments}

This research was supported in part by Grant R01HL089765 from the National Heart, Lung, and Blood Institute/ National Institutes of Health (NHLBI/NIH) (PI: Piotr Slomka). Its contents are solely the responsibility of the authors and do not necessarily represent the official views of the NHLBI. Cedars-Sinai Medical Center receives royalties for the quantitative assessment of function, perfusion, and viability, a portion of which is distributed to some of the authors of this manuscript (DB, GG, PS). We would like to thank Dr. Caroline Kilian and Arpine Oganyan for editing and proofreading the text.

\section{Abbreviations}

CAD Coronary artery disease

ML Machine learning

MPS Myocardial perfusion SPECT

TID Transient ischemic dilation

TPD Total perfusion deficit

ECG Electrocardiogram

SSS $\quad$ Summed stress score

SDS $\quad$ Summed difference score

PCI Percutaneous coronary intervention

WEKA Waikato Environment for Knowledge Analysis

\section{References}

1. Committee Members. Klocke FJ, Baird MG, et al. ACC/AHA/ASNC Guidelines for the Clinical Use of Cardiac Radionuclide Imaging-Executive Summary. Circulation. 2003; 108:1404-18. [PubMed: 12975245]

2. Gould KL, Lipscomb K, Hamilton GW. Physiologic basis for assessing critical coronary stenosis. Instantaneous flow response and regional distribution during coronary hyperemia as measures of coronary flow reserve. Am J Cardiol. 1974; 33:87-94. [PubMed: 4808557]

3. Holly TA, Abbott BG, Al-Mallah M, et al. Single photon-emission computed tomography. J Nucl Cardiol. 2010; 17:941-73. [PubMed: 20552312]

4. Danias PG, Ahlberg AW, Travin MI, et al. Visual assessment of left ventricular perfusion and function with electrocardiography-gated SPECT has high intraobserver and interobserver reproducibility among experienced nuclear cardiologists and cardiology trainees. J Nucl Cardiol. 2002; 9:263-70. [PubMed: 12032474] 
5. Amanullah AM, Berman DS, Hachamovitch R, Kiat H, Kang X, Friedman JD. Identification of severe or extensive coronary artery disease in women by adenosine technetium- $99 \mathrm{~m}$ sestamibi SPECT. Am J Cardiol. 1997; 80:132-7. [PubMed: 9230147]

6. Slomka PJ, Nishina H, Berman DS, et al. Automatic quantification of myocardial perfusion stressrest change: a new measure of ischemia. J Nucl Med. 2004; 45:183-91. [PubMed: 14960634]

7. Slomka PJ, Nishina H, Berman DS, et al. Automated quantification of myocardial perfusion SPECT using simplified normal limits. J Nucl Cardiol. 2005; 12:66-77. [PubMed: 15682367]

8. Abidov A, Bax JJ, Hayes SW, et al. Transient ischemic dilation ratio of the left ventricle is a significant predictor of future cardiac events in patients with otherwise normal myocardial perfusion SPECT. J Am Coll Cardiol. 2003; 42:1818-25. [PubMed: 14642694]

9. Johnson LL, Verdesca SA, Aude WY, et al. Postischemic stunning can affect left ventricular ejection fraction and regional wall motion on post-stress gated sestamibi tomograms. J Am Coll Cardiol. 1997; 30:1641-8. [PubMed: 9385888]

10. Arsanjani R, Xu Y, Hayes SW, et al. Comparison of fully automated computer analysis and visual scoring for detection of coronary artery disease from myocardial perfusion SPECT in a large population. J Nucl Med. 2013; 54:221-8. [PubMed: 23315665]

11. Arsanjani R, Xu Y, Dey D, et al. Improved accuracy of myocardial perfusion SPECT for detection of coronary artery disease by machine learning in a large population. J Nucl Cardiol. 2013; 20:553-62. [PubMed: 23703378]

12. Friedman J, Hastie T, Tibshirani R. Additive logistic regression: a statistical view of boosting (With discussion and a rejoinder by the authors). Ann Stat. 2000; 28:337-407.

13. Berman DS, Kiat H, Friedman JD, et al. Separate acquisition rest thallium-201/stress technetium-99m sestamibi dual-isotope myocardial perfusion single-photon emission computed tomography: a clinical validation study. J Am Coll Cardiol. 1993; 22:1455-64. [PubMed: 8227805]

14. Nishina H, Slomka PJ, Abidov A, et al. Combined supine and prone quantitative myocardial perfusion SPECT: method development and clinical validation in patients with no known coronary artery disease. J Nucl Med. 2006; 47:51-8. [PubMed: 16391187]

15. Amanullah AM, Kiat H, Friedman JD, Berman DS. Adenosine technetium-99m sestamibi myocardial perfusion SPECT in women: diagnostic efficacy in detection of coronary artery disease. J Am Coll Cardiol. 1996; 27:803-9. [PubMed: 8613606]

16. Berman DS, Kang X, Hayes SW, et al. Adenosine myocardial perfusion single-photon emission computed tomography in women compared with men. Impact of diabetes mellitus on incremental prognostic value and effect on patient management. J Am Coll Cardiol. 2003; 41:1125-33. [PubMed: 12679212]

17. Hayes SW, De Lorenzo A, Hachamovitch R, et al. Prognostic implications of combined prone and supine acquisitions in patients with equivocal or abnormal supine myocardial perfusion SPECT. $\mathrm{J}$ Nucl Med. 2003; 44:1633-40. [PubMed: 14530478]

18. Germano G, Kavanagh PB, Su HT, et al. Automatic reorientation of three-dimensional, transaxial myocardial perfusion SPECT images. J Nucl Med. 1995; 36:1107-14. [PubMed: 7769436]

19. Matsumoto N, Berman DS, Kavanagh PB, et al. Quantitative assessment of motion artifacts and validation of a new motion-correction program for myocardial perfusion SPECT. J Nucl Med. 2001; 42:687-94. [PubMed: 11337561]

20. Germano G, Kavanagh PB, Slomka PJ, Van Kriekinge SD, Pollard G, Berman DS. Quantitation in gated perfusion SPECT imaging: the Cedars-Sinai approach. J Nucl Cardiol. 2007; 14:433-54. [PubMed: 17679052]

21. Diamond GA, Forrester JS. Analysis of probability as an aid in the clinical diagnosis of coronaryartery disease. N Engl J Med. 1979; 300:1350-8. [PubMed: 440357]

22. Nishina H, Slomka PJ, Abidov A, et al. Combined supine and prone quantitative myocardial perfusion SPECT: method development and clinical validation in patients with no known coronary artery disease. J Nucl Med. 2006; 47:51-8. [PubMed: 16391187]

23. Xu Y, Arsanjani R, Clond M, et al. Transient ischemic dilation for coronary artery disease in quantitative analysis of same-day sestamibi myocardial perfusion SPECT. J Nucl Cardiol. 2012; 19:465-73. [PubMed: 22399366] 
24. Cerqueira MD, Weissman NJ, Dilsizian V, et al. Standardized myocardial segmentation and nomenclature for tomographic imaging of the heart: a statement for healthcare professionals from the Cardiac Imaging Committee of the Council on Clinical Cardiology of the American Heart Association. Circulation. 2002; 105:539-42. [PubMed: 11815441]

25. Guyon I, Elisseeff A. An Introduction to Variable and Feature Selection. J Mach Learn Res. 2003; 3:1157-82.

26. Hall M, Frank E, Holmes G, Pfahringer B, Reutemann P, Witten I. The WEKA data mining software: An update. SIGKDD Explor. 2009; 11:10-8.

27. Molinaro AM, Simon R, Pfeiffer RM. Prediction error estimation: a comparison of resampling methods. Bioinforma Oxf Engl. 2005; 21:3301-7.

28. Geisser, S. Predictive Inference: An Introduction. Chapman \& Hall; 1993. p. 31-42.

29. DeLong ER, DeLong DM, Clarke-Pearson DL. Comparing the areas under two or more correlated receiver operating characteristic curves: a nonparametric approach. Biometrics. 1988; 44:837-45. [PubMed: 3203132]

30. Patel MR, Peterson ED, Dai D, et al. Low diagnostic yield of elective coronary angiography. N Engl J Med. 2010; 362:886-95. [PubMed: 20220183]

31. Golub RJ, Ahlberg AW, McClellan JR, et al. Interpretive reproducibility of stress Tc-99m sestamibi tomographic myocardial perfusion imaging. J Nucl Cardiol. 1999; 6:257-69. [PubMed: 10385181]

32. Golub RJ, McClellan JR, Herman SD, et al. Effectiveness of nuclear cardiology training guidelines: a comparison of trainees with experienced readers. J Nucl Cardiol. 1996; 3:114-8. [PubMed: 8799236]

33. Hachamovitch R, Hayes SW, Friedman JD, Cohen I, Berman DS. Comparison of the short-term survival benefit associated with revascularization compared with medical therapy in patients with no prior coronary artery disease undergoing stress myocardial perfusion single photon emission computed tomography. Circulation. 2003; 107:2900-7. [PubMed: 12771008]

34. Hachamovitch R, Rozanski A, Shaw LJ, et al. Impact of ischaemia and scar on the therapeutic benefit derived from myocardial revascularization vs. medical therapy among patients undergoing stress-rest myocardial perfusion scintigraphy. Eur Heart J. 2011; 32:1012-24. [PubMed: 21258084] 
A

\section{Prediction of Revascularization: Stress Data}

$100.0 \%$

$90.0 \%$

$80.0 \%$

$70.0 \%$

$60.0 \%$

$50.0 \%$

$40.0 \%$

$30.0 \%$

$20.0 \%$

$10.0 \%$

$0.0 \%$
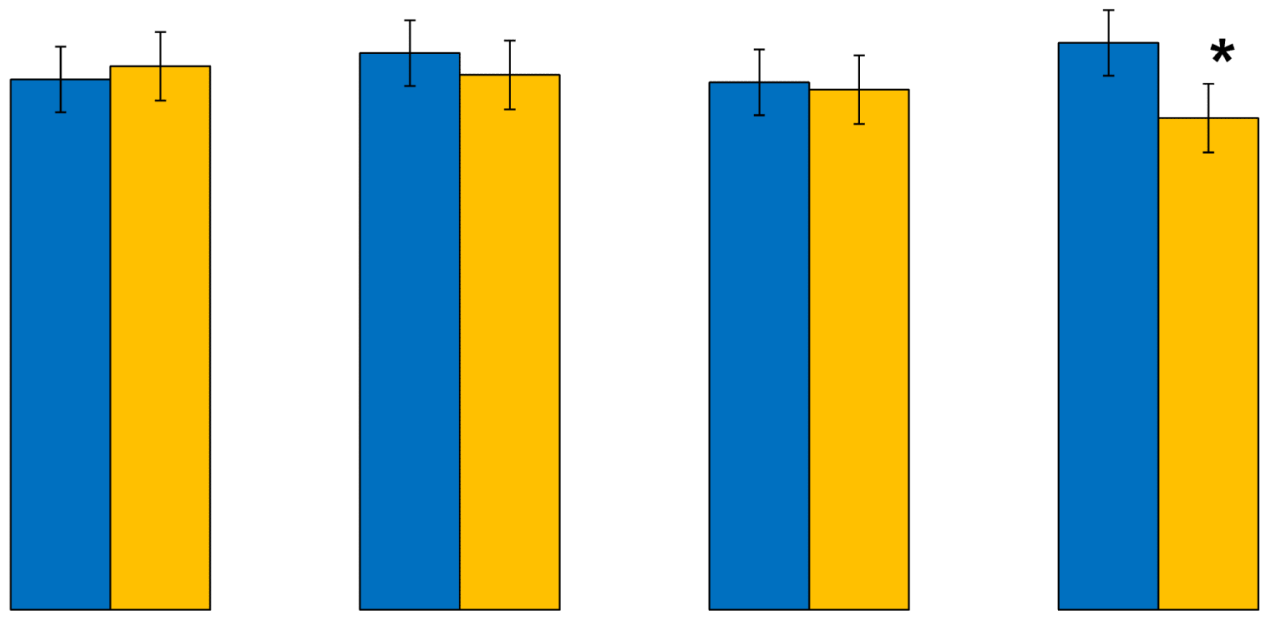

\begin{tabular}{|c|c|c|c|c|}
\hline & $M L$ & Expert 1 SSS & Expert 2 SSS & Stress TPD \\
\hline$\square$ Sensitivity \% & $72.7 \pm 4.5$ & $76.3 \pm 4.6$ & $72.3 \pm 4.6$ & $77.7 \pm 4.5$ \\
\hline$\square$ Specificity \% & $74.5 \pm 4.1$ & $73.3 \pm 4.6$ & $71.3 \pm 4.7$ & $67.4 \pm 5.0^{*}$ \\
\hline
\end{tabular}

ML: Machine Learning

SSS: Visual Summed Stress Score Using Imaging and Clinical Data TPD: Total Perfusion Deficit

${ }^{*} \mathrm{ML}$ is better $\mathrm{p}<0.05$ 


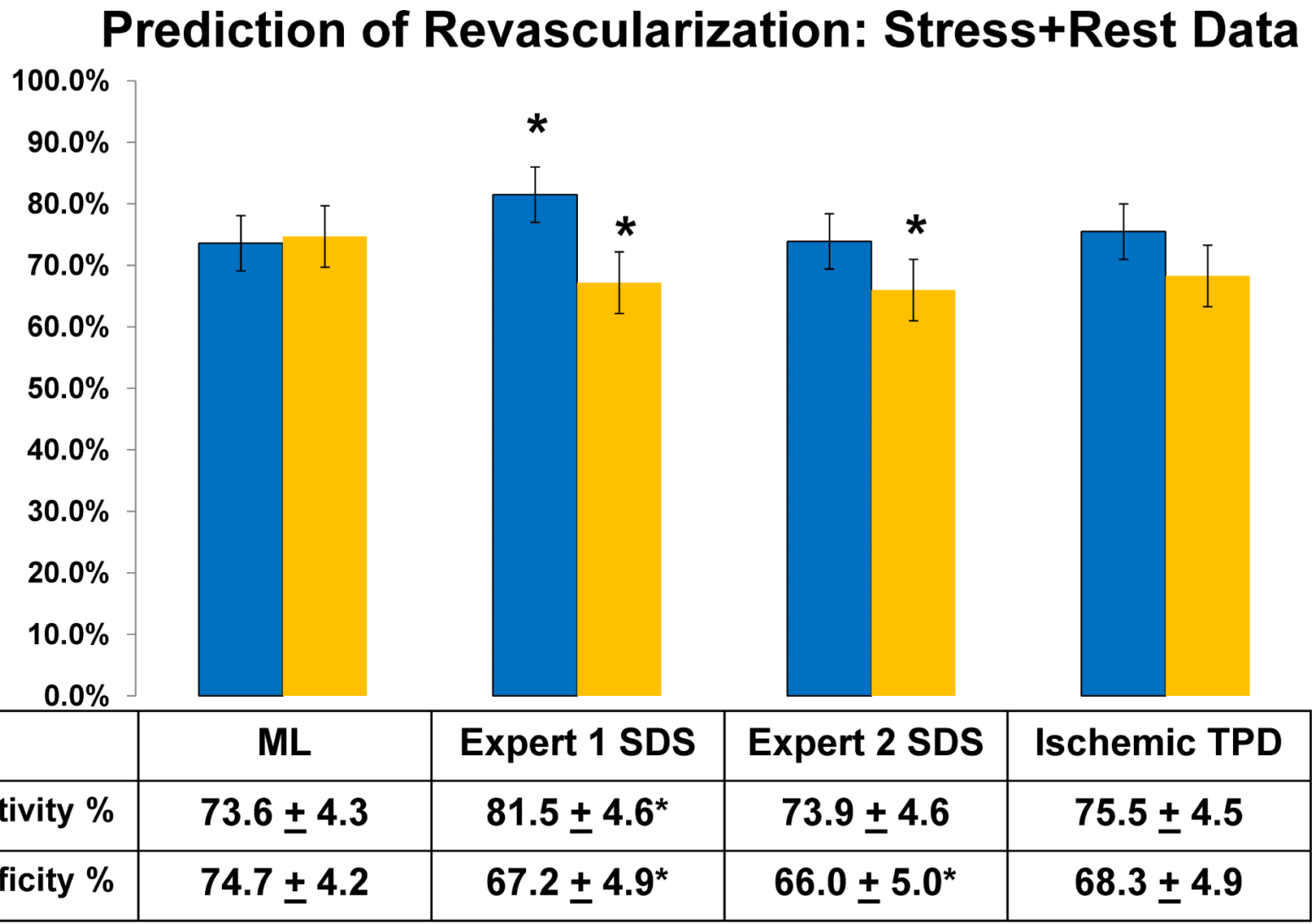

\section{ML: Machine Learning}

SDS: Visual Summed Difference Score Using Imaging and Clinical Data

Figure 1.

A) Sensitivity and specificity of ML algorithm vs combined stress supine/prone TPD and expert visual summed stress scores (SSS) and B) ML algorithm vs combined ischemic supine/prone TPD and expert visual summed difference scores (SDS) for predicting revascularization. 

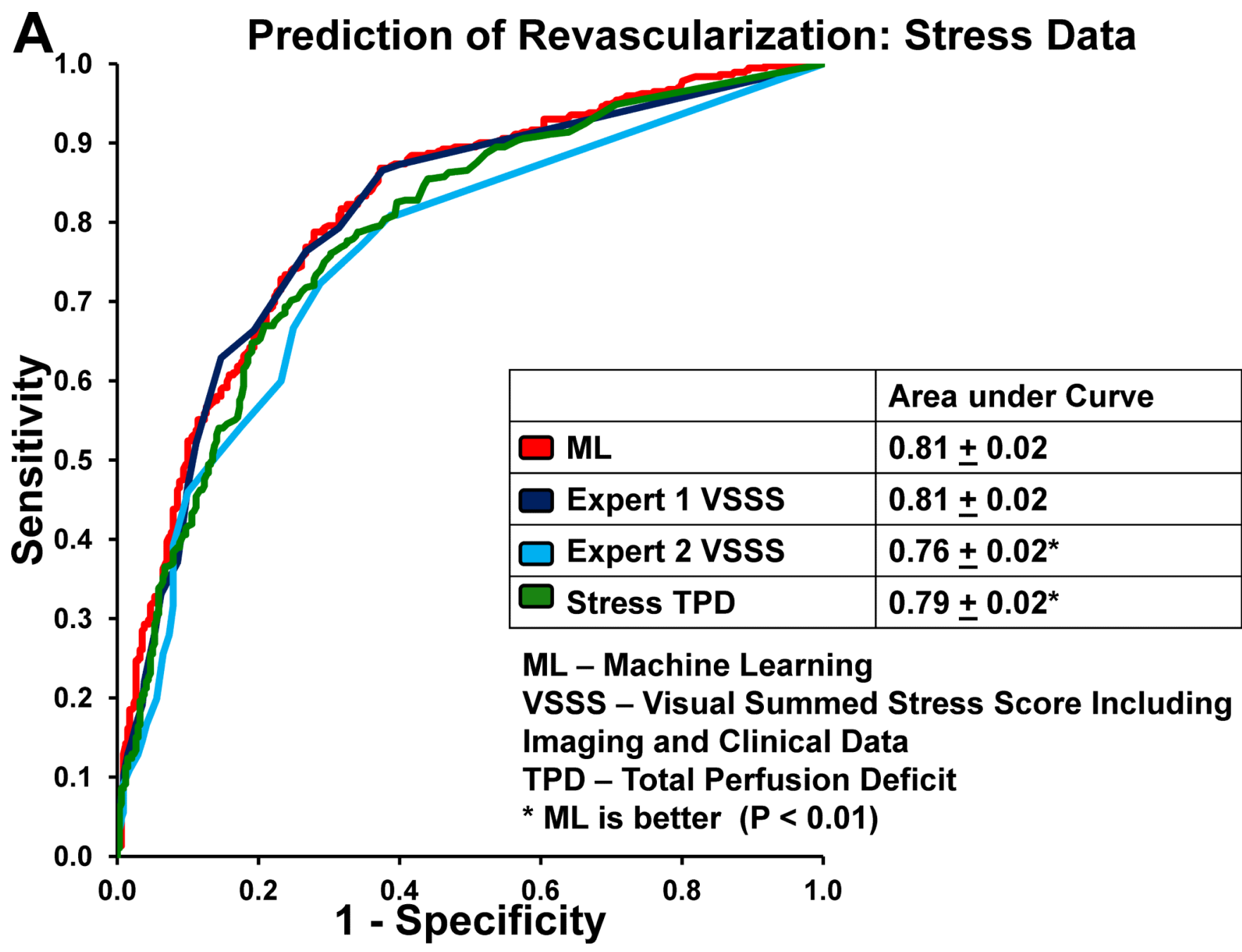


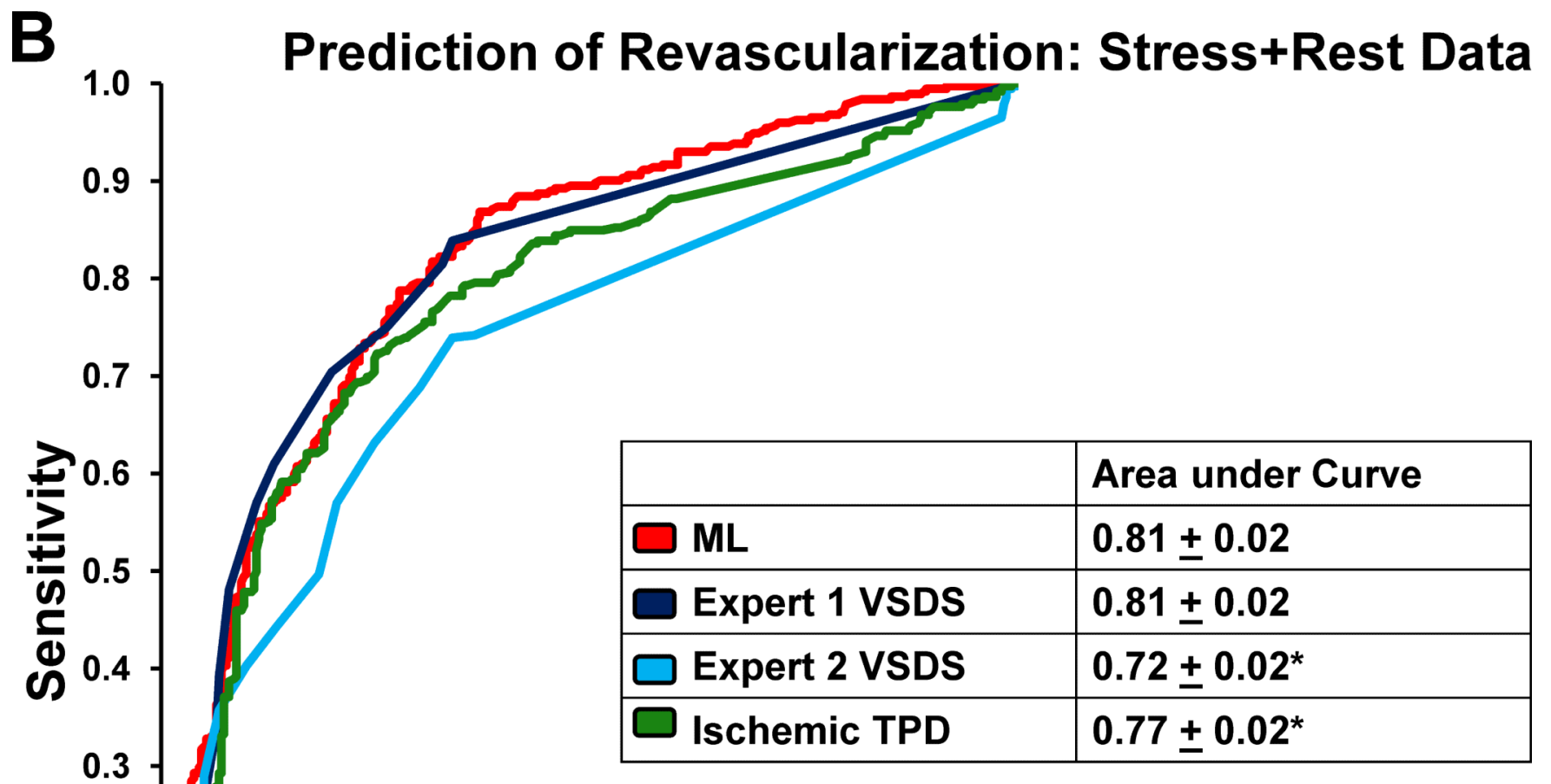

ML - Machine Learning

VSDS - Visual Summed Difference Score Including Imaging and Clinical Data TPD - Total Perfusion Deficit

0.1 ${ }^{*} M L$ is better $(P<0.01)$

0.0

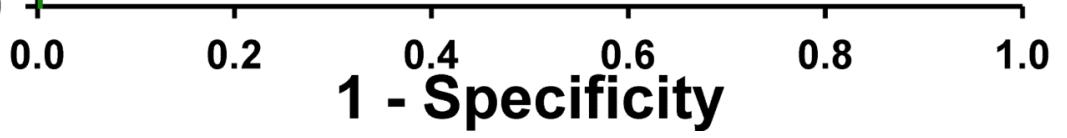

Figure 2.

A) The ROC curves comparing the ML algorithm vs combined stress supine/prone TPD and expert visual summed stress scores (SSS) and B) ML algorithm vs combined ischemic supine/prone TPD and expert visual summed difference scores (SDS) for predicting revascularization. 
Table 1

Baseline characteristics of the patients.

\begin{tabular}{|l|l|l|l|}
\hline & Non-Revascularized Group & Revascularized Group & P - Value \\
\hline Number & 341 & 372 & N/A \\
\hline Age (Years) & $62 \pm 13$ & $66 \pm 11$ & $<.01$ \\
\hline Male \% & $55 \%$ & $72 \%$ & $<.01$ \\
\hline Female \% & $45 \%$ & $28 \%$ & $<.01$ \\
\hline Diabetes Mellitus \% & $16 \%$ & $27 \%$ & $<.01$ \\
\hline Hypertension \% & $57 \%$ & $63 \%$ & $\mathbf{0 . 1 0}$ \\
\hline Hyperlipidemia \% & $48 \%$ & $56 \%$ & 0.03 \\
\hline Smoking \% & $5 \%$ & $7 \%$ & $\mathbf{0 . 2 6}$ \\
\hline Family History \% & $11 \%$ & $11 \%$ & $\mathbf{1 . 0}$ \\
\hline
\end{tabular}

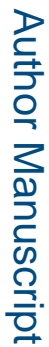

N/A = Not applicable. 


\section{Table 2}

Ranking of the features by the automated feature selection algorithm.

\begin{tabular}{|c|c|}
\hline Features & Ranking \\
\hline Stress TPD+ ${ }^{*}$ & 0.1876 \\
\hline Quantitative Stress TPD & 0.18 \\
\hline ST-Depression at Rest ${ }^{*}$ & 0.0394 \\
\hline ECG Response During Exercise* & 0.0341 \\
\hline Quantitative Rest TPD & 0.336 \\
\hline Post ECG likelihood of CAD & 0.032 \\
\hline Clinical Response During Exercise ${ }^{*}$ & 0.0305 \\
\hline Transient Ischemic Dilation (TID) & 0.0265 \\
\hline Gender ${ }^{*}$ & 0.232 \\
\hline Stress Ejection Fraction ${ }^{*}$ & 0.0188 \\
\hline End-Systolic Volume (ESV) & 0.018 \\
\hline Resting BP & 0.0158 \\
\hline History of Diabetes Mellitus ${ }^{*}$ & 0.0136 \\
\hline Age & 0 \\
\hline Heart Rate / Peak Heart rate/Peak BP (3) & 0 \\
\hline History of hypertension & 0 \\
\hline Pretest likelihood of CAD (before ECG) & 0 \\
\hline Clinical response & 0 \\
\hline Family History of CAD & 0 \\
\hline History of Hyperlipidemia & 0 \\
\hline Smoking & 0 \\
\hline Height/Weight/ Body Mass Index (3) & 0 \\
\hline Exercise Duration & 0 \\
\hline METs Achieved & 0 \\
\hline Claudication & 0 \\
\hline Symptoms at Rest & 0 \\
\hline Rest TPD+ & 0 \\
\hline Rest Ejection Fraction & 0 \\
\hline End-Diastolic Volume & 0 \\
\hline
\end{tabular}

indicates selected features. CAD - Coronary Artery Disease, METs - Metabolic Equivalent of Task. BP - Blood pressure. (number of features given in brackets if multiple) 